\title{
A novel peptide promotes human trophoblast proliferation and migration through PI3K/Akt/mTOR signaling pathway
}

\author{
Li Ling ${ }^{1}$, Xiao Yuan ${ }^{2}$, Xia Liu ${ }^{1}$, Wenjun Pei ${ }^{3}$, Ranran Li $^{1}$ \\ ${ }^{1}$ Department of Obstetrics and Gynecology, the First Affiliated Hospital of Wannan Medical College, Wuhu, China; ${ }^{2}$ Department of Ultrasound, \\ Wuwei People's Hospital, Wuhu, China; ${ }^{3}$ Anhui Province Key Laboratory of Biological Macro-Molecules Research, Wannan Medical College, \\ Wuhu, China \\ Contributions: (I) Conception and design: L Ling; (II) Administrative support: W Pei; (III) Provision of study materials or patients: R Li; (IV) \\ Collection and assembly of data: R Li, X Yuan; (V) Data analysis and interpretation: L Ling, X Yuan, R Li; (VI) Manuscript writing: All authors; (VII) \\ Final approval of manuscript: All authors. \\ Correspondence to: Ranran Li. Department of Obstetrics and Gynecology, the First Affiliated Hospital of Wannan Medical College, Wuhu. China. \\ Email: 1291071434@qq.com; Wenjun Pei. Anhui Province Key Laboratory of Biological Macro-Molecules Research, Wannan Medical College, \\ Wuhu, China. Email: $41836820 @ q q . c o m$.
}

Background: Preeclampsia (PE) is a complex pregnancy-related disease that endangers the safety of maternal and fetal. The purpose of this study is to reveal the pathogenesis of preeclampsia and discover new predictors from the perspective of peptidomics. The umbilical cord blood of PE and control group was analyzed by peptidomics. A peptide named Regulation of Proliferation Process in Preeclampsia (ROPPIP) was screened out to explore its role in the proliferation, migration and apoptosis of trophoblast cells in preeclampsia.

Methods: We compared and analyzed the umbilical cord blood of patients with PE and normal pregnant women using liquid chromatography-tandem mass spectrometry (LC-MS). hTR-8/Svneo cells cultured in vitro were divided into ROPPIP group and a disordered peptide group as control. Cell Counting Kit- 8 (CCK-8) assay, flow cytometry, Transwell chamber assays and western blot analysis were performed to detect cell proliferation, invasion, migration and apoptosis, in addition to the expression of Matrix metalloproteinase-2 (MMP2), nuclear associated antigen Ki67, B-cell lymphoma-2 (Bc12), Caspase 3, and $\beta$-actin protein.

Results: We identified 133 differential peptides. Of these, 51 were up-regulated while 82 were downregulated. the polypeptide SFGVRMATASPTDGNV with low differential expression in the serum of PE patients was selected for the study, we named the polypeptide as Regulation of Proliferation Process in PE (ROPPIP). The experiment shows that ROPPIP can up-regulate the expression levels of MMP2, Ki67, and Bcl2 in HTR-8/Svneo cells, down-regulate the expression of caspase-3, promote the proliferation and migration of HTR-8/Svneo cells and inhibit the apoptosis induced by cisplatin, the activation of the phosphatidylinositol-3-kinase/protein kinase B/mammalian target of rapamycin (PI3K/Akt/mTOR) signaling pathway may be associated with the function of ROPPIP.

Conclusions: ROPPIP promotes HTR-8/Svneo cells migration and proliferation, and inhibits apoptosis, by regulating the activation of the PI3K/AKT/mTOR signaling pathway.

Keywords: Regulation of Proliferation Process in Preeclampsia (ROPPIP); proliferation; migration; human trophoblast cells

Submitted Apr 08, 2021. Accepted for publication Jun 16, 2021.

doi: 10.21037/atm-21-2574

View this article at: https://dx.doi.org/10.21037/atm-21-2574 


\section{Introduction}

Preeclampsia (PE) is a pregnancy-specific disease, which becomes more complex with the passage of gestational weeks, and is characterized by hypertension and albuminuria after 20 weeks of pregnancy. In developing countries, it has been established as a major cause of maternal, fetal, and neonatal morbidity and mortality (1-3). This disease can also cause kidney injury, liver injury, cardio-cerebrovascular accidents, and fetal growth restriction in pregnant women (4). Currently, early termination of pregnancy is the most effective treatment modality for PE. Early intervention through the study of its pathogenesis is a new strategy for the prevention and treatment of PE (5). In the past few decades, numerous studies on the pathogenesis of $\mathrm{PE}$ have demonstrated that serum markers and long-chain non-coding Ribonucleic Acid (LncRNA), circular RNA (CircRNA), microRNA, proteomics, and genomics play an essential role in the pathogenesis of PE (6-9).

Peptide analysis is a new subject in proteomics, which is sometimes superior compared to proteomics in terms of simple structure, convenient operation, extensive research, and property stability. In a sense, polypeptides are inherited and developed from proteomics $(10,11)$. Peptides are ubiquitous in the human body, such as in organs and tissues, as well as in cells and body fluids. More importantly, peptide analysis provides us with valuable information that improves our knowledge about the synthesis, modification, and degradation of proteins, thus giving us a better understanding of the properties and molecular functions of proteins (12). Several studies have confirmed that peptides can be used in the diagnosis or treatment of a variety of diseases, including Alzheimer's disease, cancer, atherosclerosis, obesity, and diabetes (13-19). It has been identified that there are 35 statistically different polypeptides in the placentas of PE patients and normal pregnancies (20). At the same time, it has been found that unique peptides derived from $\alpha$-antitrypsin, type I collagen, type III collagen, and urinary modulin can be used as early diagnostic indicators of PE (21). Furthermore, it has been emphasized that SERPINA1 peptide in urine remains one of the most promising polypeptide markers of PE (21). Taken together, these studies suggest that peptides may be a useful potential target for the prevention and treatment of PE.

Our team is committed to discovering biomarkers with potential application value in PE. By collecting the umbilical cord blood of PE patients and women with normal pregnancies for peptide analysis, we uncovered a new peptide isolated from the protein SORL1 (Sortilinrelated receptor). SORL1 is a Protein Coding gene. Diseases associated with SORL1 include Early-Onset, Autosomal Dominant Alzheimer Disease and Alzheimer Disease. SORL1 Plays a role in renal ion homeostasis, Stimulates, via the N-terminal ectodomain, the proliferation and migration of smooth muscle cells. This may promote extracellular matrix proteolysis and hence facilitate cell migration (22). By acting on the migration of intimal smooth muscle cells, may accelerate intimal thickening following vascular injury (22). However, at present, there is a dearth of information about the relationship between SORL1-derived peptides and PE. In order to perform this study, we hereby named the SORL1-derived peptides Regulation of Proliferation Process in Preeclampsia (ROPPIP) in order to explore its mechanism and its role in trophoblasts. We present the following article in accordance with the MDAR reporting checklist (available at https://dx.doi.org/10.21037/atm-21-2574).

\section{Methods}

\section{Sample collection, peptide extraction, and LC-MS/MS analysis}

Umbilical cord blood was collected from PE and normal pregnancy patients hospitalized in the obstetrics department of the First Affiliated Hospital of Wannan Medical College, and stored in a refrigerator at $4{ }^{\circ} \mathrm{C}$ overnight before analysis. All procedures performed in this study involving human participants were in accordance with the Declaration of Helsinki (as revised in 2013). The study was approved by institutional ethics committee of the First Affiliated Hospital of Wannan Medical College, Wuhu, China (No. 202133) and informed consent was taken from all the patients. Serum samples were centrifuged at a rate of 5,000 g at $4{ }^{\circ} \mathrm{C}$ for 5 minutes, and the supernatant was collected. Next, mixed protease inhibitors were added to the samples and preserved at $-80{ }^{\circ} \mathrm{C}$, followed by centrifugation of the sample at a rate of $12,000 \mathrm{~g}$ at $4{ }^{\circ} \mathrm{C}$ for 15 minutes. After centrifugation, the rich protein was removed using a filter membrane with a molecular weight cut-off (MWCO) of $10 \mathrm{kDa}$. The polypeptides were then collected, purified, recovered, and freeze-dried. Subsequently, freeze-dried peptides were further dissolved and filtered, desalted, and freeze-dried.

Afterward, $20 \mu \mathrm{g}$ samples were marked with a $5 \mu \mathrm{L}$ Tandem Mass Tag (TMT) reagent according to the manufacturer's instructions (TMT6-plex Label Reagent, 
Thermo Fisher Science, USA). The labeled samples were analyzed using Nanoflow LC (Thermo Fisher Science, USA) combined with mass spectrometry. We employed LTQ-Orbitrap Velos mass spectrometer (Thermo Fisher Science, USA) for analysis, while all data were collected, converted, and analyzed using MassLynx analyzer software (Waters, USA). Additionally, the GO and pathway analysis (https://david.ncifcrf.gov/) were used to predict the possible functions of the identified peptides. The precursor proteins of different peptides were assessed using functional cluster analysis using the Database for Annotation Visualization and Integrated Discovery (DAVID) online website (https:// david.ncifcrf.gov), whereas the physical and chemical properties of the peptides were evaluated using ExPASY analysis (http://web.expasy.org/protparam/).

\section{Synthesis of peptides}

The ROPPIP (SFGVRMATASPTDGNV) used in this study, which were comprised of 16-amino-acid sequences derived from the SORL1, ROPPIP and scramble peptide (MSRATPTDGNFAGVSV) were synthesized by Shanghai Scientific Peptide Biological Technology Co., Ltd (Shanghai, China). All peptides N-terminals were coupled with fluoresceine isothiocyanate (FITC) dye for labeling.

\section{Cell culture}

Human trophoblast cells (HTR-8/Svneo) were acquired from Wuhan Procell Life Technology Co., Ltd. (catalog number: CL-0599, Wuhan, China). The cells were cultured with Roswell Park Memorial Institute (RPMI) 1640 media (Sigma, USA) supplemented with $10 \%$ fetal bovine serum (FBS), $100 \mathrm{U} / \mathrm{mL}$ penicillin, and $100 \mathrm{mg} / \mathrm{mL}$ streptomycin double-antibody, which was placed in a constant temperature and humidity incubator containing $5 \%$ carbon dioxide $\left(\mathrm{CO}_{2}\right)$ at $37^{\circ} \mathrm{C}$. The culture media was changed every day and the cells were passaged when they reached $90 \%$ confluence. HTR-8/Svneo cells were administrated with $100 \mu \mathrm{M}$ FITC-ROPPIP and incubated for $1 \mathrm{~h}$ at $37^{\circ} \mathrm{C}$ in the dark, then imaged with a Confocal laser scanning microscopy (magnification, $\times 200$ ).

\section{Cell counting kit-8 (CCK-8)}

The CCK-8 (RiboBio, China) was used to determine cell proliferation, as per the manufacturer's instructions. HTR8/Svneo cells were seeded in 96 -well plates at a density of
$4 \times 10^{3}$ cells per well and grown overnight. Next, the HTR8/Svneo cells were co-cultured with ROPPIP (10, 50, $100 \mu \mathrm{mol} / \mathrm{L}$ ) for $0,24,48$, and $72 \mathrm{~h}$. The optical density $(\mathrm{OD})$ value was measured at $450 \mathrm{~nm}$ using a microplate reader (BioTek, USA).

\section{Flow cytometry}

HTR-8/Svneo cells were induced with cisplatin and then treated with ROPPIP, ROPPIP treatment with their density adjusted to $5 \times 10^{5} / \mathrm{mL}$. Thereafter, $0.5 \mathrm{~mL}$ cell suspension and $1.25 \mu \mathrm{L}$ AnnexinV-FITCs (Nanjing KGI Biotechnology Co., Ltd., Nanjing) were mixed and placed in the dark at room temperature for $10 \mathrm{~min}$. The tube was then centrifuged at 1,000 revolutions per min for $5 \mathrm{~min}$. The cells were then suspended in $0.2 \mathrm{~mL}$ precooled binding buffer, and $5 \mu \mathrm{L}$ of propidium iodide (PI) was subsequently added. The analysis was then immediately performed using flow cytometry.

\section{Wound healing assay}

HTR-8/Svneo cells (at a population of $2 \times 10^{5}$ cells/well) were inoculated into a six-well plate. Next, the cell layer was scratched with a sterile pipette tip and treated with ROPPIP. The scrambled peptide was washed in a serumfree medium. Scratched areas were examined under a microscope at $48 \mathrm{~h}$, while the rate of gap closure was quantified using Image J software (Rawak Software, Inc. Germany).

\section{Transwell assays}

The cells were collected after ROPPIP treatment, resuspended in 300 microL of serum-free medium and seeded in the upper chamber of a transwell, while a culture medium (700 microL) containing 10\% fetal bovine serum was added to the lower chamber. After incubation, the cells were fixed with methanol, stained with $0.1 \%$ crystal violet and counted.

\section{Western blotting}

HTR8/SVneo cells were lysed with RadioImmunoprecipitation Assay (RIPA) buffer (Beyotime Institute of Biotechnology, China), and the total protein was extracted. The protein concentration was measured using the bicinchoninic acid (BCA) method (Beyotime Institute 
of Biotechnology, China). After processing with the loading buffer, proteins $(30 \mu \mathrm{g})$ were subjected to electrophoresis with a $10 \%$ polyacrylamide gel, and were subsequently transferred to polyvinylidene fluoride (PVDF) (EMD Millipore, USA) membranes. After blocking with $5 \%$ fatfree milk for $1 \mathrm{~h}$ at room temperature, membranes were incubated with primary antibodies at $4{ }^{\circ} \mathrm{C}$ overnight.

The primary antibodies used in this study were as follows: Matrix metalloproteinase-2 (MMP2) (1:2,000, CST, USA), Ki-67 (1:2,000, CST, USA), B-cell lymphoma-2 (Bcl2) $(1: 2,000$, CST, USA), caspase-3 (1:2,000, CST, USA), phosphorylated (p)- Phosphatidylinositol 3-kinase(pPI3K) (1:2,000, BIOSS , USA), PI3K (1:2,000, CST, USA), phosphorylated (p)-mammalian target of rapamycin (p-mTOR) (1:2,000, Abcam, England), mTOR (1:2,000, CST, USA), protein kinase B (AKT) (1:2,000, CST, USA), and $\beta$-actin $(1: 5,000$, ProteinTech Group, USA). After washing three times with Tris-Buffered Saline and Tween 20 (TBST) containing $0.1 \%$ Tween-20 (10 min every time) at room temperature, membranes were incubated with secondary antibody, goat anti-rabbit immunoglobin $\mathrm{G}$ (IgG) horseradish peroxidase (1:2,000; cat. no. ab205718; Abcam), for $1 \mathrm{~h}$ at room temperature. After washing again with TBST, the immunoblots were visualized using chemiluminescence reagents (EMD Millipore, USA), while the grey value of immunoblots was semi-quantified using Image J software (v1.52a; National Institutes of Health).

\section{Statistical analysis}

GraphPad Prism 7 software (GraphPad Software, Inc., USA) was used for statistical analysis and data plotting. Quantitative data were expressed as the mean \pm standard deviation (SD). Data from two groups were compared using a two-tailed Student's $t$-test, whereas multiple group comparisons were determined using one-way analysis of variance (ANOVA) and Bonferroni's multiple comparison test. $\mathrm{P}<0.05$ was considered statistically significant. All experiments were performed three times using independent samples, with six replications each time.

\section{Results}

\section{Differentially expressed peptides derived from the PE and control groups}

A total of 2,103 non-redundant peptides were identified from the umbilical cord blood of the PE and normal pregnancy groups using LC-MS/MS analysis. In addition, our results revealed that there were 133 differentially expressed peptides, of which, 51 peptides were upregulated, while 82 were down-regulated (fold change $\geq 2.0, \mathrm{P}<0.05$ ) (Figure $1 A, B$ ). Findings of the GO analysis revealed that these peptides were functionally involved in various biological activities, including cell proliferation, cell cycle, apoptosis, cell cycle phase, and protein binding, and were closely related to cell proliferation, apoptosis, and migration (Figure 1C). Among the cluster genes associated with cell proliferation in the GO analysis, and based on the criteria of high mass spectrum (MS) signal abundance and small intra-group and large inter-group differences, three peptides from SORL1 with low expression in PE patients were selected for further study.

\section{Analysis of the basic characteristics of peptides}

We subsequently examined the basic biological characteristics of the three peptides from SORL1 using the ExPASy website (http://web.expasy.org/protparam). We then selected SFGVRMATASPTDGNV as the study object in terms of the characteristics of hydrophobicity, lipophilicity, and easy entry into cells. Its basic biological characteristics are summarized in Figure 2A, which were named ROPPIP after labeling with a green fluorescent dye. We observed that ROPPIP could enter HTR8/Svneo cells and further into the nucleus (Figure 2B).

\section{ROPPIP inbibited apoptosis and promoted the proliferation and migration of HTR-8/Svneo cells}

To further explore the potential functions of ROPPIP peptides, we added chemically-synthesized ROPPIP peptides and non-functional scrambled peptides as a control into HTR-8/Svneo cells to detect the effects of ROPPIP on cell proliferation and apoptosis. Our results showed that ROPPIP promoted the proliferation of HTR-8/Svneo cells in a dose-dependent manner (Figure $3 A$ ). Moreover, we used PI/Annexin V staining and flow cytometry to quantify the effect of peptides on cell apoptosis. ROPPIP can inhibit the apoptosis of HTR-8/Svneo cells induced by cisplatin (Figure 3B). Notably, ROPPIP exerted the most significant effect at $100 \mu \mathrm{M}$. Therefore, a concentration of $100 \mu \mathrm{M}$ was selected as the appropriate concentration for further study. we examined the effect of ROPPIP on the migration and invasion of trophoblast cells. After $48 \mathrm{~h}$ of ROPPIP treatment, we noted that ROPPIP significantly 
A
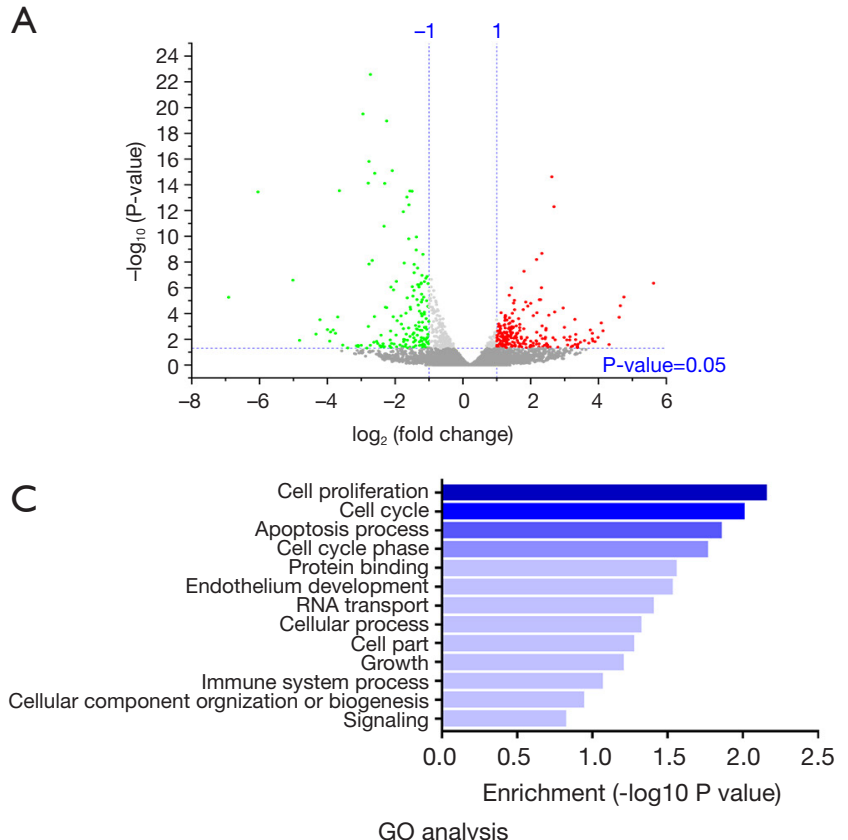

B

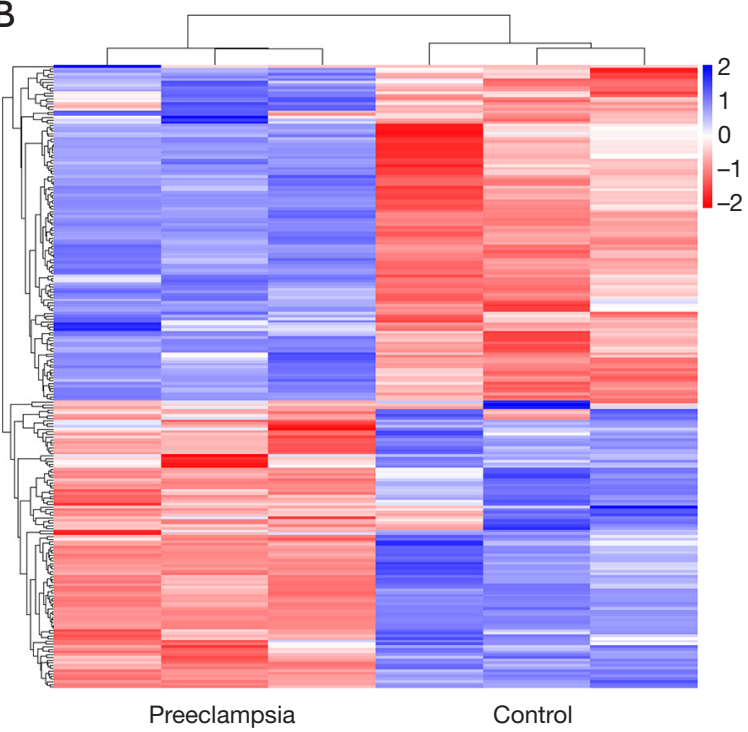

Figure 1 Serum samples from PE and normal pregnancy patients collected for peptidome analysis. The differential peptides with fold change $\geq 2.0$ and $\mathrm{P}<0.05$ were included in the volcano (A), heat map (B), and GO $(\mathrm{C})$ analyses. Three differentially low-expressed peptides (derived from SORL1) were selected from the cluster genes related to cell proliferation in the GO analysis, while three differentially lowexpressed peptides (derived from SORL1) were selected in PE patients.

\begin{tabular}{|c|c|c|c|c|c|c|c|}
\hline Peptidesequence & Protein name & MW & $\mathrm{PI}$ & Half-life & Instability index & Aliphatic index & GRAVY \\
\hline $\begin{array}{l}\text { SFGVRMATA } \\
\text { SPTDGNV }\end{array}$ & SORL1 & 1609.77 & 5.55 & $1.9 \mathrm{~h}$ & 39.24 & 48.75 & -0.013 \\
\hline $\begin{array}{l}\text { LASKTNVYIS } \\
\text { SSA }\end{array}$ & SORL1 & 1340.50 & 8.59 & $5.5 \mathrm{~h}$ & 21.5 & 97.69 & 0.269 \\
\hline $\begin{array}{l}\text { GGDVEARLE } \\
\text { GELV }\end{array}$ & SORL1 & 1343.46 & 4.00 & $30 \mathrm{~h}$ & 12.92 & 112.31 & -0.146 \\
\hline $\begin{array}{l}\text { SEDLSLEVC } \\
\text { VP }\end{array}$ & SORL1 & 1190.33 & 3.57 & $1.9 \mathrm{~h}$ & 54.76 & 123.64 & 0.436 \\
\hline
\end{tabular}

B

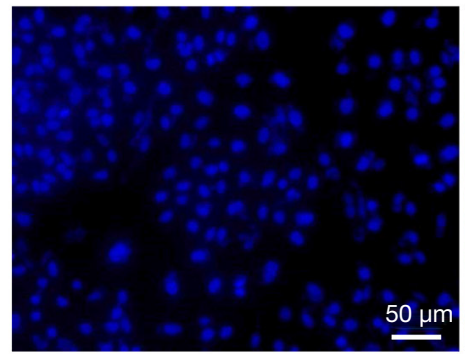

DAPI

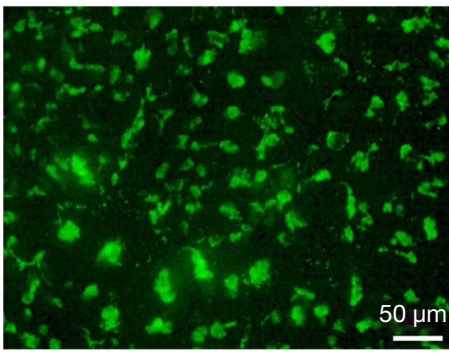

ROPPIP

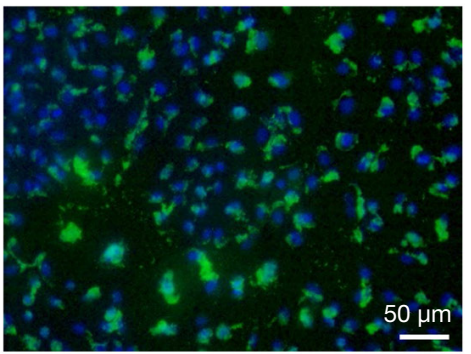

Merge

Figure 2 Analysis of physical and chemical properties of peptides screened through the ExPASy online website. (A) Three differentially lowexpressed peptides in patients with PE (derived from SORL1) were selected from the cluster genes related to cell proliferation in the GO analysis. (B) The typical fluorescence images of FITC-labeled ROPPIP incubated with HTR-8/Svneo cells for $1 \mathrm{~h}$ following DAPI staining showed that ROPPIP exhibited characteristics of penetrating cell and nuclear localization [100]. 
A

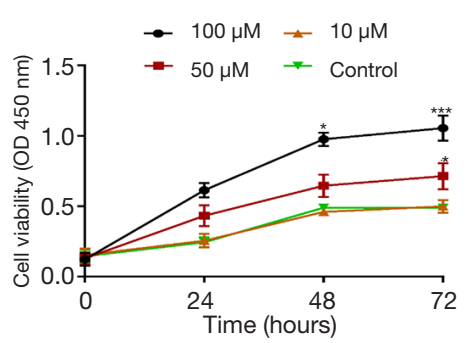

C

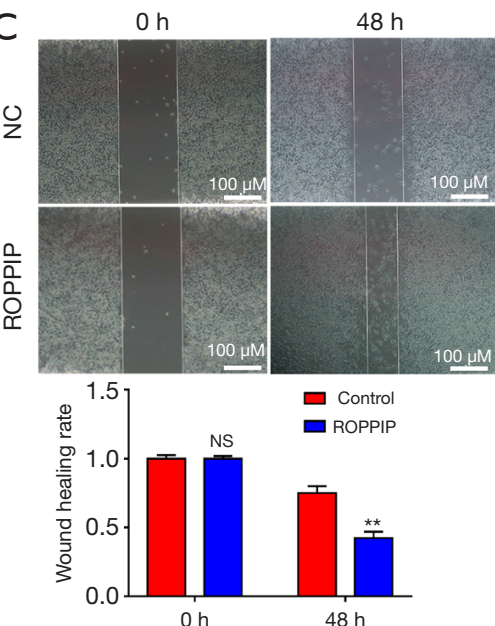

B
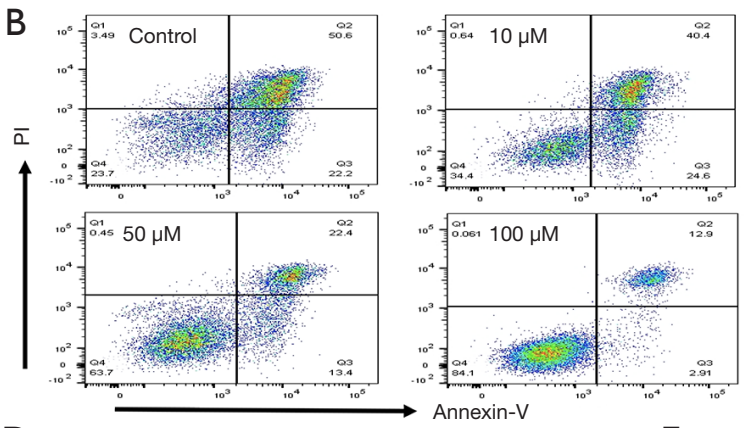

E

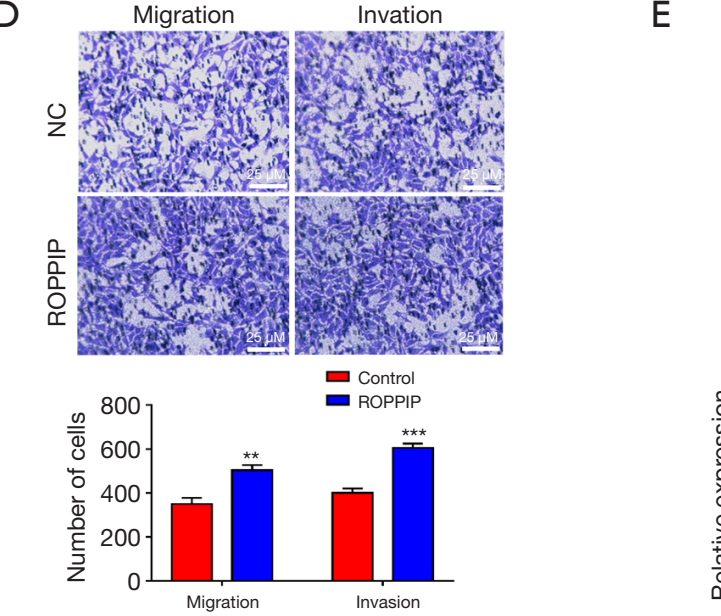

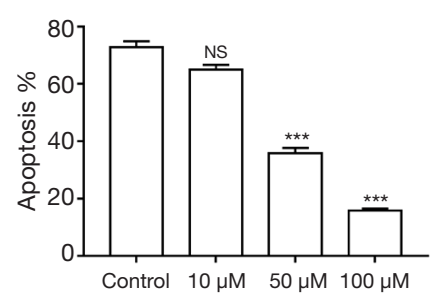

Control ROPPIP
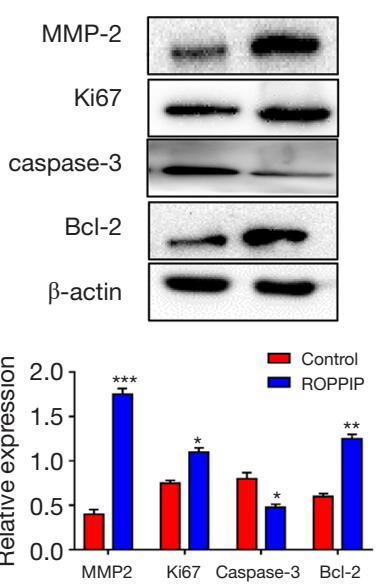

Figure 3 ROPPIP promoted the proliferation and migration of HTR-8/Svneo cells, and inhibited apoptosis. (A) ROPPIP promoted the proliferation of HTR-8/Svneo cells in a dose-dependent manner. (B) ROPPIP inhibited the apoptosis of HTR-8/Svneo cells and its statistical graph. (C) Representative images of wound healing captured at 0 and $48 \mathrm{~h}$ after cell scratching, and the wound healing rate was calculated. (D) Transwell assay revealed that ROPPIP promotes the migration and invasion of HTR-8/Svneo cells and its statistical graph. Dyeing with crystal violet, the magnification is 100x. (E) ROPPIP affected protein expression of MMP-2, Ki-67, caspase-3, and Bcl-2 in HTR-8/Svneo cells. Compared with control and its statistical graph. ${ }^{* * *} \mathrm{P}<0.001,{ }^{* *} \mathrm{P}<0.01,{ }^{*} \mathrm{P}<0.05$; NS, no significance.

promoted the migration and invasion of HTR-8/Svneo cells (Figure 3C,D) and up-regulated the expression of MMP-2 (Figure $3 E$ ). The findings of western blotting demonstrated that the expression of Ki-67 increased following ROPPIP treatment. ROPPIP could down-regulate the expression of caspase-3, while the Bcl-2 protein exhibited an opposite pattern (Figure 3E).

\section{ROPPIP promoted activation of the PI3K/AKT/mTOR signaling patbway in HTR-8/Svneo cells in vitro}

Pathway analysis results showed that the protein precursors of these differential peptides were involved in the PI3K/ AKT/mTOR, p38 mitogen-activated protein kinase (MAPK), cell cycle, and other signaling pathways (Figure 4A). Recent studies have confirmed that key proteins in thyroid hormone signaling pathways, including PI3K, AKT, and mTOR, can participate in the pathogenesis of PE (22-24). Furthermore, our findings, based on western blotting experiments, showed that the phosphorylation levels of PI3K, AKT, and mTOR increased after ROPPIP treatment (Figure $4 B, C, D)\left({ }^{*}: \mathrm{P}<0.05, \mathrm{P}<0.01, \mathrm{P}<0.001\right)$. This suggests that the activation of the $\mathrm{PI} 3 \mathrm{~K} / \mathrm{AKT} / \mathrm{mTOR}$ signaling pathway may be associated with the function of ROPPIP.

\section{Discussion}

Hypertensive disorders, such as $\mathrm{PE}$, which complicate pregnancy, continue to be a leading cause of maternal and neonatal morbidity and mortality. PE has been shown to cause multiple organ function damage, including elevated blood pressure, nervous system symptoms, pulmonary 
A

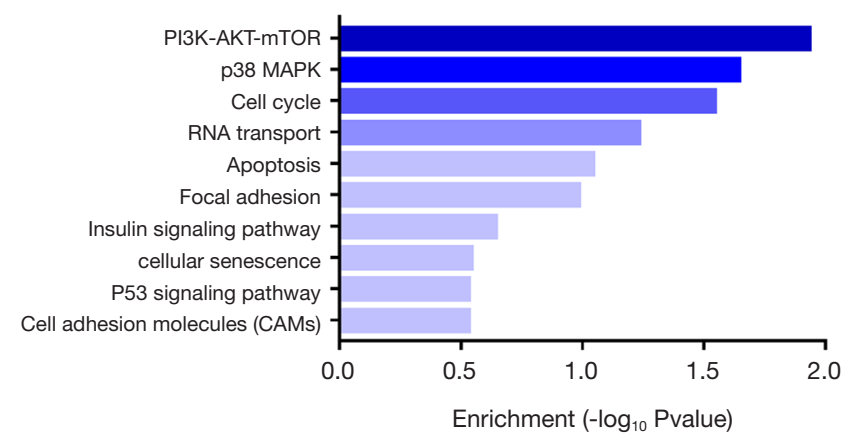

C

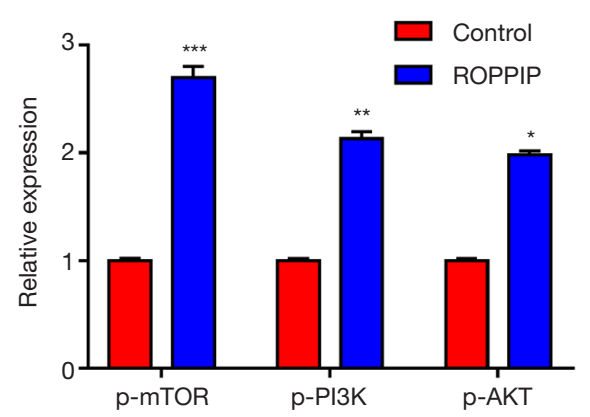

B
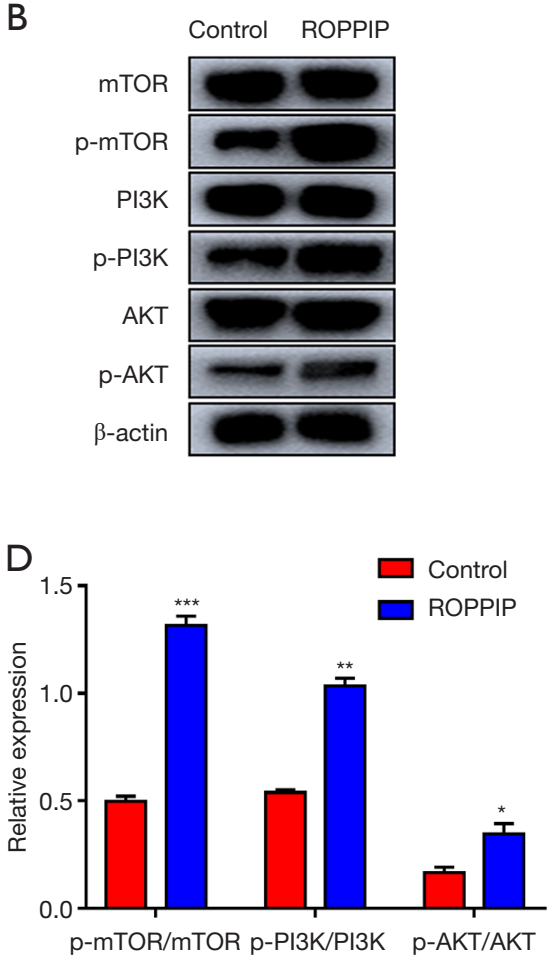

Figure 4 Pathway analysis. (A) KEGG Pathway analysis. (B) ROPPIP promoted the activation of the PI3K/AKT/mTOR signaling pathway in HTR-8/SVneo cells. Compared with control and its statistical graph (C,D), ${ }^{* *} \mathrm{P}<0.001,{ }^{* *} \mathrm{P}<0.01,{ }^{*} \mathrm{P}<0.05$.

edema, acute renal injury, thrombocytopenia, and liver dysfunction. Recent statistics have estimated that $3 \%$ of pregnant women develop PE (23), In developed countries, pregnant women who died of preeclampsia accounted for $16.1 \%$ of the deaths (24). In another study, it was reported that the overall incidence of PE increased from $3.4 \%$ in 1980 to $3.8 \%$ in 2010 , with an increase in the incidence of severe cases in all age groups (23). This increased incidence may be attributed to multiple factors. Currently, studies on the pathogenesis of $\mathrm{PE}$ have received substantial research attention from a number of researchers.

$\mathrm{PE}$ is characterized by widespread endothelial damage and dysfunction. It is a systemic syndrome originating from the placenta, which usually begins with insufficient invasion of trophoblast cells, resulting in severe endothelial dysfunction during pregnancy. Multiple studies have established that $\mathrm{PE}$ accounts for approximately $18 \%$ of all maternal deaths worldwide and $40 \%$ and global fetal mortality (25-27). Notably, this disease is a serious complication of pregnancy that increases maternal and perinatal morbidity and mortality (28). Thus far, numerous researchers have paid serious attention to the pathogenesis and pathological changes of PE. However, the specific factors causing PE remain largely unknown (29), and require further clarification. It is widely believed that early detection and intervention can greatly improve the perinatal outcome of mothers and babies.

Identification of PE using traditional monitoring methods is predominately achieved by detecting blood pressure and urinary protein levels, which fluctuate significantly with the changes of a variety of external environments and numerous other uncontrollable factors. With this in mind, there is an urgent need for the development of a more reliable and effective method to detect PE. Polypeptides refer to natural low molecular weight (LMW) peptides and protein hydrolysate fragments with molecular weight less of than $10 \mathrm{kDa}(30)$. Recent advances in mass spectrometry have enabled the direct analyses of hundreds of LMW peptides that were previously difficult to detect, especially in human serum. Most of these small molecular peptides are fragments of macromolecular precursor proteins, which may reflect the results of protein-protein interactions in the molecular pathogenesis. Interestingly, new disease markers are expected to be identified in small molecular 
polypeptides, such as polypeptide group analysis of human sera from normal and preeclamptic pregnancies. Previous studies have shown that the analysis of serum polypeptide biomarkers may be valuable for the diagnosis of PE (31).

Using polypeptide analysis, we herein identified different polypeptides in the serum of $\mathrm{PE}$ and normal pregnancy patients, some of which are derived from known precursor proteins related to $\mathrm{PE}$, such as apolipoprotein (APO) L1, flexible goal adjustment (FGA), and that peptides of a1-antitrypsin (SERPINA1), which are associated with acute inflammation and defense response. The APO-A4, APO-C3, APO-E, and APOL1 proteins are related to lipid metabolism. However, despite extensive study on the functions of precursor proteins, little is known about the functions of these peptides, including their possible biological activities. In a study by Starodubtseva et al. (21), neuropeptide analysis was performed in 79 patients with $\mathrm{PE}$, chronic pregnancy-induced hypertension, and normal pregnancy. Their findings indicated that peptides derived from SERPINA1 can resist hypoxia, reduce trophoblast invasion, and participate in the pathogenesis of PE. Also, urinary SERPINA1-derived peptides could be used to assess the severity of PE. Taken together, the above results indicate that the study of polypeptides may provide a novel direction for the prevention and treatment of PE.

In this study, we also analyzed the peptides of the umbilical cord blood of $\mathrm{PE}$ and normal pregnancy patients. Based on a fold change $>2$ and $\mathrm{P}<0.05$, we subsequently screened the polypeptide, ROPPIP, which was derived from SORL1, to detect its effect on the biological behavior of trophoblasts. Remarkably, our in vitro experiments showed that ROPPIP could promote the proliferation of HTR-8/Svneo cells, inhibit apoptosis, and increase the ability of cell migration.

It is well established that MMP is secreted by trophoblast cells, thus playing a pivotal role in trophoblast invasion and placental formation $(32,33)$, in which MMP2 is closely linked with trophoblast invasion (34). At the same time, it has been argued that the proliferation-related protein, Ki67, and apoptosis-related proteins, caspase-3 and Bcl-2, are all involved in the pathogenesis of $\mathrm{PE}$ (35). In this study, we found that ROPPIP could promote the migration and proliferation of trophoblasts, inhibit their apoptosis, upregulate the expression of MMP2, Ki-67, and Bcl-2, and down-regulate the expression of caspase- 3 .

The pathogenesis of $\mathrm{PE}$ is a complex pathological process, involving a variety of molecular and signal pathways. Previous studies have shown that in PE, the phosphorylation levels of PI3K and mTOR decrease in response to oxidative stress $(36,37)$. In this study, we identified that the expression of p-PI3K, p-AKT and p-mTOR were up-regulated following ROPPIP treatment, implying that the activation of the PI3K/AKT/mTOR signaling pathway may be correlated with the function of ROPPIP in promoting trophoblast proliferation and migration. Previous studies have revealed that PI3K inhibitor LY294002 was used to inhibit the proliferation and migration of damaged endothelial cells promoted by PDCC4 (Peptide derived from complement C4 A chain) in PE (38). Therefore, the PI3K inhibitor is possibly related to the biological functions of trophoblasts regulated by the ROPPIP/PI3K/AKT/mTOR pathway.SORL1 stimulates, via the $\mathrm{N}$-terminal ectodomain, the proliferation and migration of smooth muscle cells, possibly by increasing cell surface expression of the urokinase receptor uPAR/ PLAUR (22). The down-regulation of uPAR can inhibit the $\mathrm{PI} 3 \mathrm{~K} / \mathrm{AKT} / \mathrm{mTOR} / \mathrm{HIF} 1 \alpha$ signaling pathway induced by the antagonist peptide (39). Therefore, whether the peptide ROPPIP derived from SORL1 affects the PI3K/AKT/ mTOR signaling pathway through uPAR and thus plays a role in preeclampsia will be the content of our further research.

In summary, the results of this study demonstrate that ROPPIP promotes trophoblast cell migration and proliferation, and inhibits apoptosis, by regulating the activation of the PI3K/AKT/mTOR signaling pathway. This suggests that ROPPIP may be a potential prospect in the treatment of PE. However, the underlying regulatory mechanism of ROPPIP needs to be clarified so as to provide a new target for the early diagnosis and treatment of PE.

\section{Acknowledgments}

Funding: The present study was supported by grants from the Special Fund for Clinical Research of Wu Jieping Medical Foundation (No. 320.6750.17325), and the Anhui University Natural Science Research Project (No. KJ2020A0612).

\section{Footnote}

Reporting Checklist: The authors have completed the MDAR reporting checklist. Available at https://dx.doi. org/10.21037/atm-21-2574

Data Sharing Statement: Available at https://dx.doi. org/10.21037/atm-21-2574 
Conflicts of Interest: All authors have completed the ICMJE uniform disclosure form (available at https://dx.doi. org/10.21037/atm-21-2574). The authors have no conflicts of interest to declare.

Ethical Statement: The authors are accountable for all aspects of the work in ensuring that questions related to the accuracy or integrity of any part of the work are appropriately investigated and resolved. All procedures performed in this study involving human participants were in accordance with the Declaration of Helsinki (as revised in 2013). The study was approved by institutional ethics committee of the First Affiliated Hospital of Wannan Medical College, Wuhu, China (No. 202133) and informed consent was taken from all the patients.

Open Access Statement: This is an Open Access article distributed in accordance with the Creative Commons Attribution-NonCommercial-NoDerivs 4.0 International License (CC BY-NC-ND 4.0), which permits the noncommercial replication and distribution of the article with the strict proviso that no changes or edits are made and the original work is properly cited (including links to both the formal publication through the relevant DOI and the license). See: https://creativecommons.org/licenses/by-nc-nd/4.0/.

\section{References}

1. Mol BWJ, Roberts CT, Thangaratinam S, et al. Preeclampsia. Lancet 2016;387:999-1011.

2. Venkatesha S, Toporsian M, Lam C, et al. Soluble endoglin contributes to the pathogenesis of preeclampsia. Nat Med 2006;12:642-9.

3. Levine RJ, Lam C, Qian C, et al. Soluble endoglin and other circulating antiangiogenic factors in preeclampsia. $\mathrm{N}$ Engl J Med 2006;355:992-1005.

4. Powe CE, Levine RJ, Karumanchi SA. Preeclampsia, a disease of the maternal endothelium: the role of antiangiogenic factors and implications for later cardiovascular disease. Circulation 2011;123:2856-69.

5. Peter Stein T, Scholl TO, Schluter MD, et al. Oxidative stress early in pregnancy and pregnancy outcome. Free Radic Res 2008;42:841-8.

6. Jia R, Li J, Rui C, et al. Comparative Proteomic Profile of the Human Umbilical Cord Blood Exosomes between Normal and Preeclampsia Pregnancies with HighResolution Mass Spectrometry. Cell Physiol Biochem 2015;36:2299-306.
7. Pillar N, Yoffe L, Hod M, et al. The possible involvement of microRNAs in preeclampsia and gestational diabetes mellitus. Best Pract Res Clin Obstet Gynaecol 2015;29:176-82.

8. Zhang YG, Yang HL, Long Y, et al. Circular RNA in blood corpuscles combined with plasma protein factor for early prediction of pre-eclampsia. BJOG 2016;123:2113-8.

9. Qian Y, Lu Y, Rui C, et al. Potential Significance of Circular RNA in Human Placental Tissue for Patients with Preeclampsia. Cell Physiol Biochem 2016;39:1380-90.

10. Ivanov VT, Yatskin ON. Peptidomics: a logical sequel to proteomics. Expert Rev Proteomics 2005;2:463-73.

11. Dallas DC, Guerrero A, Parker EA, et al. Current peptidomics: applications, purification, identification, quantification, and functional analysis. Proteomics 2015;15:1026-38.

12. Qian Y, Zhang L, Rui C, et al. Peptidome analysis of amniotic fluid from pregnancies with preeclampsia. Mol Med Rep 2017;16:7337-44.

13. Hwang JT, Park IJ, Shin JI, et al. Genistein, EGCG, and capsaicin inhibit adipocyte differentiation process via activating AMP-activated protein kinase. Biochem Biophys Res Commun 2005;338:694-9.

14. Gao Y, Zhou Y, Xu A, et al. Effects of an AMP-activated protein kinase inhibitor, compound $\mathrm{C}$, on adipogenic differentiation of 3T3-L1 cells. Biol Pharm Bull 2008;31:1716-22.

15. Zhang T, Sawada K, Yamamoto N, et al. 4-Hydroxyderricin and xanthoangelol from Ashitaba (Angelica keiskei) suppress differentiation of preadiopocytes to adipocytes via AMPK and MAPK pathways. Mol Nutr Food Res 2013;57:1729-40.

16. Wang X, You L, Cui X, et al. Evaluation and optimization of differentiation conditions for human primary brown adipocytes. Sci Rep 2018;8:5304.

17. Tormos KV, Anso E, Hamanaka RB, et al. Mitochondrial complex III ROS regulate adipocyte differentiation. Cell Metab 2011;14:537-44.

18. Wang X, Mandal AK, Saito H, et al. Arsenic and chromium in drinking water promote tumorigenesis in a mouse colitis-associated colorectal cancer model and the potential mechanism is ROS-mediated $\mathrm{Wnt} / \beta$-catenin signaling pathway. Toxicol Appl Pharmacol 2012;262:11-21.

19. Shen D, Li Y, Wang X, et al. A novel peptide suppresses adipogenic differentiation through activation of the AMPK pathway. Biochem Biophys Res Commun 2019;510:395-402.

20. Kononikhin AS, Starodubtseva NL, Bugrova AE, et al. 
An untargeted approach for the analysis of the urine peptidome of women with preeclampsia. J Proteomics 2016;149:38-43.

21. Starodubtseva N, Nizyaeva N, Baev O, et al. SERPINA1 Peptides in Urine as A Potential Marker of Preeclampsia Severity. Int J Mol Sci 2020;21:914.

22. Zhu Y, Bujo H, Yamazaki H, et al. LR11, an LDL receptor gene family member, is a novel regulator of smooth muscle cell migration. Circ Res 2004;94:752-8.

23. Ananth CV, Keyes KM, Wapner RJ. Pre-eclampsia rates in the United States, 1980-2010: age-period-cohort analysis. BMJ 2013;347:f6564.

24. Khan KS, Wojdyla D, Say L, et al. WHO analysis of causes of maternal death: a systematic review. Lancet 2006;367:1066-74.

25. Anderson UD, Olsson MG, Kristensen KH, et al. Review: Biochemical markers to predict preeclampsia. Placenta 2012;33 Suppl:S42-7.

26. Wang A, Rana S, Karumanchi SA. Preeclampsia: the role of angiogenic factors in its pathogenesis. Physiology (Bethesda) 2009;24:147-58.

27. Dai X, Cai Y. Down-regulation of microRNA let-7d inhibits the proliferation and invasion of trophoblast cells in preeclampsia. J Cell Biochem 2018;119:1141-51.

28. Ghulmiyyah L, Sibai B. Maternal mortality from preeclampsia/eclampsia. Semin Perinatol 2012;36:56-9.

29. Shah DM. Preeclampsia: new insights. Curr Opin Nephrol Hypertens 2007;16:213-20.

30. Tirumalai RS, Chan KC, Prieto DA, et al. Characterization of the low molecular weight human serum proteome. Mol Cell Proteomics 2003;2:1096-103.

31. Wen Q, Liu LY, Yang T, et al. Peptidomic Identification of Serum Peptides Diagnosing Preeclampsia. PLoS One

Cite this article as: Ling L, Yuan X, Liu X, Pei W, Li R. A novel peptide promotes human trophoblast proliferation and migration through PI3K/Akt/mTOR signaling pathway. Ann Transl Med 2021;9(12):981. doi: 10.21037/atm-21-2574 2013;8:e65571.

32. Yeh CC, Chao KC, Huang SJ. Innate immunity, decidual cells, and preeclampsia. Reprod Sci 2013;20:339-53.

33. Zhu JY, Pang ZJ, Yu YH. Regulation of trophoblast invasion: the role of matrix metalloproteinases. Rev Obstet Gynecol 2012;5:e137-43.

34. Sakowicz A, Lisowska M, Biesiada L, et al. Association of Maternal and Fetal Single-Nucleotide Polymorphisms in Metalloproteinase (MMP1, MMP2, MMP3, and MMP9) Genes with Preeclampsia. Dis Markers 2018;2018:1371425.

35. Cao C, Li J, Li J, et al. Long Non-Coding RNA Uc.187 Is Upregulated in Preeclampsia and Modulates Proliferation, Apoptosis, and Invasion of HTR-8/SVneo Trophoblast Cells. J Cell Biochem 2017;118:1462-70.

36. Zhang X, Li Q, Jiang W, et al. LAMA5 promotes human umbilical vein endothelial cells migration, proliferation, and angiogenesis and is decreased in preeclampsia. J Matern Fetal Neonatal Med 2020;33:1114-24.

37. Chen J, Yue C, Xu J, et al. Downregulation of receptor tyrosine kinase-like orphan receptor 1 in preeclampsia placenta inhibits human trophoblast cell proliferation, migration, and invasion by PI3K/AKT/mTOR pathway accommodation. Placenta 2019;82:17-24.

38. Xue L, Xie K, Wu L, et al. A novel peptide relieves endothelial cell dysfunction in preeclampsia by regulating the PI3K/mTOR/HIF $1 \alpha$ pathway. Int J Mol Med 2021;47:276-88.

39. Laurenzana A, Chillà A, Luciani C, et al. uPA/uPAR system activation drives a glycolytic phenotype in melanoma cells. Int J Cancer 2017;141:1190-200.

(English Language Editor: A. Kassem) 